\title{
2016 Revision to the WHO classification of acute myeloid leukemia
}

\author{
Ming Hong, Guangsheng He \\ Department of Hematology, The First Affiliated Hospital of Nanjing Medical University, \\ Jiangsu Province Hospital, Nanjing 210029, Jiangsu Province, China
}

\section{ACUTE MYELOID \\ LEUKEMIA WITH RECURRENT GENETIC ABNORMALITIES}

The subtype "acute myeloid leukemia (AML) with inv(3) (q21.3q26.2) or t(3;3)(q21.3q26.2); RPN1-EVI1" in the nomenclature of the WHO 2008 classification is now renamed as "AML with inv(3)(q21.3q26.2) or t(3;3)(q21.3q26.2); GATA2, MECOM" because the inv(3)(q21.3q26.2) or $\mathrm{t}(3 ; 3)$ (q21.3q26.2) was not found to represent a fusion gene, but to reposition a distal GATA2 enhancer to activate MECOM expression, and simultaneously confer GATA2 haploinsufficiency. Stefen et al. analyzed 41 samples using targeted next generation sequencing (NGS), functional genomics and genome-engineering, with confirmed EVI1 overexpression and harboring an inv(3)(q21.3q26.2) or a $\mathrm{t}(3 ; 3)(\mathrm{q} 21.3 \mathrm{q} 26.2)$, including 38 primary bone marrow samples from patients, that is AML $(n=33)$, chronic myeloid leukemia - blast crisis (CML-BC, $n=2$ ), and myelodysplastic syndromes (MDS, $n=3$ ), as well as three cell lines (MUTZ-3, molm-1 and UCSD-AML1). ${ }^{[1]}$ The data showed that $\operatorname{inv}(3) / t(3 ; 3)$ chromosomal rearrangements cause dysregulation of two specific AML predisposition genes, EVI1 and GATA2, by aberrant activity of a single enhancer element in its ectopic chromatin environment, without the formation of an oncogenic fusion product. The identified enhancer appears to originally control the transcription of $110 \mathrm{~kb}$ distant GATA2 gene at $3 q 21$, and not the nearby gene RPN1. Overexpression of EVI1 is caused by the inappropriate transcriptional control of the ectopic GATA2 regulatory element, while GATA2 transcriptional impairment results from the removal of that same enhancer from its genomic origin. Thus, it has been verified that not RPN1, but rather the GATA2 locus is the source of the ectopic enhancer activating EVI1 in leukemia harboring inv(3)/t(3;3). Moreover, GATA2 is a critical hematopoietic stemness factor and aberrant EVI1 activation argues for a primitive HSC defect in this subtype of AML. Rewiring of parts of the GATA2 and EVI1 domains led to a reduction of GATA2 expression levels. Since primitive hematopoietic precursors will be particularly susceptible to disturbances of GATA2 homeostasis, GATA2 deficiency may provide the right spatiotemporal context for EVI1 oncogene activation, that is in the right cell at the right stage of differentiation for subsequent malignant transformation. Notably, GATA2 expression levels in primary inv(3)/t(3;3) AML cases and cell lines $(n=78)$ were found to be significantly reduced as compared to controls (213 AML patients). In addition, monoallelic GATA2 expression was found as a consequence of GATA2 inactivation on the rearranged allele in cases with inv(3) or $\mathrm{t}(3 ; 3)$. Thus, the inversion/translocation event in $\operatorname{inv}(3) / t(3 ; 3)$ malignancies reorganizes an originally upstream regulatory element of the GATA2 domain, causing reduced and functional haploin sufficiency of GATA2, which represent a well-established underlying cause of MDS/AML.

The PML-RARA fusion, which plays a central role in the pathogenesis of this characteristic subtype of disease, may result from complex cytogenetic rearrangements other than $\mathrm{t}(15 ; 17)(\mathrm{q} 24.1 ; \mathrm{q} 21.2)$. Thus, acute promyelocytic leukemia (APL) with this fusion is renamed as APL with PMLRARA in WHO 2016 classification. 
Two new provisional categories of AML have been added to the WHO classification, AML with BCR-ABL1 and AML with mutated RUNX1. The BCR/ABL1 fusion gene is rarely in de novo AML. Array Comparative Genomic Hybridization (aCGH) was used to study six $\mathrm{Ph}(+)$ AML searching for specific genomic profiles. ${ }^{[2]}$ In this study, the loss of IKZF1 and/or CDKN2A genes, the hallmark of $\mathrm{Ph}(+) \mathrm{ALL}$, was surprisingly found to be recurrent in $\mathrm{Ph}(+) \mathrm{AML}$ and accompanied cryptic deletions within the immunoglobulin (IGH) and T cell receptor genes (TCR). The latter two losses have been shown to be a part of the "hot spot" genome imbalances associated with BCR/ABL1 positive pre-B lymphoid phenotype in CML and $\mathrm{Ph}(+)$ ALL. While the biological and clinical significance of this specific genome signature remains to be uncovered, the deletion of antigen receptor genes (IGH, TCR), IKZF1 and/or CDKN2A may support a diagnosis of de novo AML versus blast phase of CML. With respect to the molecular characterization of $\mathrm{Ph}(+)$ AML, a study from MD Anderson Cancer Centers screened $\mathrm{Ph}(+) \mathrm{AML}$ and 5 CML-BP (blastic phase) patients initially presented in $\mathrm{BP}$ for mutations in 14 genes. The data showed that $\mathrm{Ph}+$ AML is distinct from CML-BP. All the patients with $\mathrm{Ph}(+)$ AML were negative for $A B L 1$ and other gene mutations. One (20\%) CML-BP patients had ABL1 mutation; two of $9(22 \%) \mathrm{Ph}(+)$ AML patients had NPM1 mutations, while no patients had NPM1 mutations.

The analyses of RUNX1 mutations (RUNX1mut) in 147 patients with normal karyotype or noncomplex chromosomal imbalances showed that RUNX1mut were most frequent in FAB classification M0 (65.2\%) followed by M2 (32.4\%) and M1 (30.2\%). ${ }^{[3]}$ Considering cytogenetics, RUNX1mut were most frequent in cases with +13 (27 of $30,90 \%$ ), whereas frequencies were similar in other cytogenetic groups such as MLL gene (19.7\%), FLT3ITD (16.3\%), and NRAS mutations (9.5\%). Patients with RUNX1mut had shorter OS and EFSl compared with RUNX1 wild-type cases (median, 378 days $v$ s. not reached, $P=0.003$; and median, $285 v$ s. 450 days, $P=0.003$, respectively). Multivariate analysis showed independent prognostic relevance for OS for RUNX1 mut $(P=0.029)$, FLT3-ITD $(P=0.003)$, age $(P<0.001)$, and white blood cell count $(P<0.002)$. To determine the association of RUNX1 mutations with therapeutic outcome in younger and older patients with primary cytogenetically normal acute myeloid leukemia (CN-AML), 175 younger ( $<60$ years) and 225 older ( $\geq 60$ years) patients treated with intensive cytarabine/anthracycline-based first-line therapy on Cancer and Leukemia Group B protocols were centrally analyzed for RUNX1 mutations and for established prognostic gene mutations. ${ }^{[4]}$ The results demonstrated that RUNX1 mutations are twice as common in older than younger patients with $\mathrm{CN}-\mathrm{AML}$ and negatively impact the outcome in both age groups. RUNX1-mutated patients had lower complete remission rates $(P=0.005$ in younger; $P=0.006$ in older) and shorter disease-free survival $(P=0.058$ in younger; $P<0.001$ in older), overall survival $(P=0.003$ in younger; $P<0.001$ in older $)$, and event-free survival $(P<0.001$ for younger and older) than RUNX1 wild-type patients. RUNX1-mutated blasts have molecular features of primitive hematopoietic and lymphoid progenitors, potentially leading to novel therapeutic approaches.

The finding that the improved prognosis associated with AML with mutated CEBPA, which are seen in 5\% to $14 \%$ of AML, is associated with biallelic, but not single, mutations of the gene has resulted in a change in that disease definition to require biallelic mutations. Most AMLs with CEBPA mutations simultaneously carry 2 mutations ( $\left.C E B P A^{\text {double-mut }}\right)$, usually biallelic, whereas single heterozygous mutations ( $\left.C E B P A^{\text {single-mut }}\right)$ are less frequently seen. Wouters et al. ${ }^{[5]}$ identified a subset of 41 CEBPA mutant cases from a cohort of 598 newly diagnosed AML cases (28CEBP $A^{\text {double-mut }}$ and $13 C E B P A^{\text {single-mut }}$ cases). $C E B P A^{\text {double-mut }}$ associated with a unique gene expression profile, as well as favorable overall and event-free survival, retained in multivariable analysis that included cytogenetic risk, FLT3-ITD and NPM1 mutation, white blood cell count, and age. In contrast, $C E B P A^{\text {single-mut }}$ AMLs did not express a discriminating signature and could not be distinguished from wild-type cases as regards clinical outcome. These results demonstrated significant underlying heterogeneity within CEBPA mutation positive AML with prognostic relevance. Additionally, due to the lack of prognostic significance of multilineage dysplasia in patients without MDS-associated cytogenetic findings and with a mutation of NPM1 or biallelic mutation of CEBPA, these mutations now supersede the presence of multilineage dysplasia in the classification. In other words, the presence of multilineage dysplasia alone will not classify a case as AML with myelodysplasia-related changes when a mutation of NPM1 or biallelic mutation of CEBPA is present. $\operatorname{Del}(9 \mathrm{q})$ has been removed as a defining cytogenetic abnormality for AML with myelodysplasia-related changes because of its association with NPM1 or biallelic CEBPA mutations and its apparent lack of prognostic significance in those settings.

\section{AML, NOT OTHERWISE SPECIFIED}

The subcategory of acute erythroid leukemia, erythroid/ myeloid type (previously defined as a case with $\geq 50 \% \mathrm{BM}$ erythroid precursors and $\geq 20 \%$ myeloblasts among nonerythroid cells) has been removed from the AML category. In the new classification, myeloblasts are always counted as a percentage of total marrow cells and the majority 
of such cases have $<20 \%$ total blast cells and are now classified as MDS (usually MDS with excess blasts). This change was based on the close biologic relationship of erythroid/myeloid type acute erythroid leukemia to MDS in terms of its clinical presentation, morphologic features and genetic abnormalities, as well as the low reproducibility of non-erythroid blast counts and an attempt to achieve uniformity in expressing the blast percentages across all myeloid neoplasms. Cases with $\geq 50 \%$ or more erythroid cells and $\geq 20 \%$ total myeloblasts usually meet the criteria for AML with myelodysplasia-related changes and should be diagnosed as such; cases with $\geq 20 \%$ total myeloblasts not meeting criteria for AML with myelodysplasia-related changes or AML with recurrent genetic abnormalities should be categorized as one of the other subtypes of AML, NOS.

\section{Conflict of Interest}

None declared.

\section{REFERENCES}

1. Groschel S, Sanders MA, Hoogenboezem R, de Wit E, Bouwman BA, Erpelinck C, et al. A single oncogenic enhancer rearrangement causes concomitant EVI1 and GATA2 deregulation in leukemia. Cell 2014;157:369-81.

2. Nacheva EP, Grace CD, Brazma D, Gancheva K, Howard-Reeves J, Rai $\mathrm{L}$, et al. Does BCR/ABL1 positive acute myeloid leukemia exist? Br J Haematol 2013;161:541-50.

3. Schnittger S, Dicker F, Kern W, Wendland N, Sundermann J, Alpermann T, et al. RUNX1 mutations are frequent in de novo AML with noncomplex karyotype and confer an unfavorable prognosis. Blood 2011;117:2348-57.

4. Mendler JH, Maharry K, Radmacher MD, Mrozek K, Becker H, Metzeler KH, et al. RUNX1 mutations are associated with poor outcome in younger and older patients with cytogenetically normal acute myeloid leukemia and with distinct gene and MicroRNA expression signatures. J Clin Oncol 2012;30:3109-18.

5. Wouters BJ, Löwenberg B, Erpelinck-Verschueren CAJ, van Putten WLJ, Valk PJM, Delwel R. Double CEBPA mutations, but not single CEBPA mutations, define a subgroup of acute myeloid leukemia with a distinctive gene expression profile that is uniquely associated with a favorable outcome. Blood 2009;113:3088-91.

How to cite this article: Hong M, He G. 2016 Revision to the WHO classification of acute myeloid leukemia. J Transl Intern Med 2017; 5: 69-71. 